\title{
The Best Parameterization of Initial Value Problem for Mixed Difference-Differential Equation ${ }^{\star}$
}

\author{
A. Kopylov and E. Kuznetsov \\ Moscow Aviation Institute, Volokolamskoe shosse 4, \\ 125993 Moscow, Russia \\ akopylov@nes.ru, Kuznetsov@mai.ru
}

\begin{abstract}
We develop an approach to the numerical integration of initial value problem for mixed difference-differential equations that are differential with respect to one argument and difference with respect to others. Preliminary reduction of the problem to a set of Cauchy problems for systems of ordinary differential equations depending on a parameter affords to state it as the problem of the continuing the solution with respect to the best continuation parameter, namely, the integral curve length. This statement has numerous advantages over the usual statement. Namely, the right-hand sides of the transformed system remain bounded even if right-hand sides of the original system become infinite at some points.
\end{abstract}

\section{Introduction}

The general type of mixed functional-differential equations (MFDE) [1] is

$$
x^{(l+1)}(s, t)=f\left(t, s, x_{t s}, x_{t s}^{\prime}, \ldots, x_{t s}^{(l)}\right)
$$

Here $a<s<b,-\infty<a<b<\infty, 0<t<\infty$. The ranges of values of $x$ and $f$ lie in $\mathbb{R}^{n}$; the $i$-th order derivative of $x_{t s}$ with respect to $\mathrm{t}$ is denoted as $x_{t s}^{(i)}, i=1, \ldots, l+1 ; x_{t s}(\vartheta, \xi)=x(t+\vartheta, s+\xi),-g \leq \vartheta \leq 0,|\xi| \leq h ; h, g \geq 0$.

A particular case of general MFDE of the form

$F\left(x^{\prime}(s, t), x^{\prime}\left(s+h_{1}, t\right), \ldots, x^{\prime}\left(s+h_{k}, t\right), x(s, t), x\left(s+h_{1}, t\right), \ldots, x\left(s+h_{k}, t\right)\right)=0$,

where $x: \mathbb{R}^{2} \rightarrow \mathbb{R}, s \in \Omega \subset \mathbb{R}^{n}, h_{i} \in \mathbb{Z}^{n}, i=1, \ldots, k, k \in \mathbb{N}$, is called the mixed difference-differential equation (MDDE).

Despite equations of this type have a lot of different applications in ecology, biology and physics they have been studied systematically not for a long time. The exhaustive review of applications and studies is presented in [2], the general theory of such equations is given in [1]. Paper [3] deals with boundary value problem for MDDEs which appear from variational problems. Paper [4]

\footnotetext{
* Supported by the Russian Foundation for Basic Research, project $N^{o}$ 01-01-00038
} 
develops an algorithm of numerical integration of boundary value problem for linear MDDE based on finite difference method. This work presents an approach to numerical integration of initial value problem for MDDE transformed to the best argument. The efficiency of the approach is shown on test example.

\section{Initial Value Problem for Mixed Difference-Differential Equation}

Let consider mixed difference-differential equation

$$
R_{1}(t, s) \frac{\partial}{\partial t} x(t, s)=R_{2}(t, s) g(x(t, s))+f(t, s), \quad((t, s) \in Q),
$$

with boundary condition

$$
x(t, s)=0, \quad\left((t, s) \in(0, T) \times\left(\mathbb{R}^{n} \backslash \Omega\right),\right.
$$

and initial condition

$$
x(0, s)=\varphi(s), \quad(s \in \Omega) .
$$

Here $Q=(0, T) \times \Omega, \Omega \subset \mathbb{R}^{n}$ is a bounded domain with piecewise-smooth boundary, $0<T<\infty, f(t, s): Q \rightarrow \mathbb{R}$ and $g(x): \mathbb{R} \rightarrow \mathbb{R}$;

$$
\left(R_{\nu} y\right)(t, s)=\sum_{h \in H} a_{\nu}^{h}(t, s) y(t, s+h), \quad \nu=1,2
$$

where $H$ is an additive Abelian group of integer vectors, $a_{\nu}^{h}(t, s): Q \rightarrow \mathbb{R}$, $\nu=1,2, \quad h \in H$.

Suppose that $f(t, s), \quad a_{\nu}^{h}(t, s), \quad \nu=1,2, h \in H$ are continuous functions with respect to $t$ and piecewise-smooth with respect to $s \in Q$ and $g(x)$ is a continuous function in $\mathbb{R}$.

Let $D \subset \mathbb{R}^{n+1}$ be a bounded domain. Denote by $\tilde{C}^{1,0}(D)$ a set of functions $y(t, s): D \rightarrow \mathbb{R}$ that are continuously differentiable with respect to the first argument for almost all values of $s$ and piecewise-smooth with respect to the second argument. As it was done in [5], introduce operators $I_{Q}, P_{Q}, R_{Q}$ as follows:

$I_{Q}: \tilde{C}^{1,0}(Q) \rightarrow \tilde{C}^{1,0}\left((0, T) \times \mathbb{R}^{n}\right)$ extends a function from the space $\tilde{C}^{1,0}(Q)$ with zero to $\left((0, T) \times \mathbb{R}^{n}\right) \backslash Q$; to $Q$;

$P_{Q}: \tilde{C}^{1,0}\left((0, T) \times \mathbb{R}^{n}\right) \rightarrow \tilde{C}^{1,0}(Q)$ restricts a function from $\tilde{C}^{1,0}\left((0, T) \times \mathbb{R}^{n}\right)$

$R_{\nu Q}: \tilde{C}^{1,0}(Q) \rightarrow \tilde{C}^{1,0}(Q), \nu=1,2$ are difference operators given by

$$
R_{\nu Q}=P_{Q} R_{\nu} I_{Q}, \quad(\nu=1,2) .
$$

Definition 1. A function $x(t, s) \in \tilde{C}^{1,0}(Q)$ is the solution to problem (1)-3), if it satisfies the following equation

$$
R_{1 Q}(t, s) \frac{\partial}{\partial t} x(t, s)=R_{2 Q}(t, s) g(x(t, s))+f(t, s), \quad((t, s) \in Q),
$$

and initial condition 


$$
x(t, s)=\varphi(s), \quad((t, s) \in\{0\} \times \Omega),
$$

for almost all $s \in \Omega$.

Note that it is the definition of difference operators $R_{\nu Q}$ that affords to rearrange equation (1) and boundary condition (2) to equation (4).

It has been proved (see [5]) that $\Omega \backslash\left(\bigcup_{h \in H}(\partial \Omega+h)\right)$ breaks into classes of subdomains $\Omega_{i j}$ (here and further we use $i=1, \ldots, I$ for a class number and $j=1, \ldots, J_{i}$ is a subdomain number in class $i$ ). Subdomains $\Omega_{i_{1} j_{1}}$ and $\Omega_{i_{2} j_{2}}$ belong to the same class iff there is a vector $h \in H$ such that $\Omega_{i_{1} j_{1}}=\Omega_{i_{2} j_{2}}+h$.

Denote

$$
Q_{i j}=(0, T) \times \Omega_{i j} .
$$

It is evident that

$$
\bar{Q}=\bigcup_{i=1}^{I} \bigcup_{j=1}^{J_{i}} \bar{Q}_{i j} \text { and } Q_{i_{1} j_{1}} \bigcap Q_{i_{2} j_{2}}=\emptyset,\left(i_{1}, j_{1}\right) \neq\left(i_{2}, j_{2}\right) .
$$

Rearrange problem (4), (5). Let an isomorphism

$$
U_{i}: \tilde{C}^{1,0}\left(\bigcup_{j=1}^{J_{i}} Q_{i j}\right) \rightarrow \tilde{C}_{J_{i}}^{1,0}\left(Q_{i 1}\right)
$$

be given by

$$
\left(U_{i} x\right)_{j}(t, s)=x\left(t, s+h_{i j}\right), \quad\left((t, s) \in Q_{i 1}\right),
$$

where $h_{i j}$ such that $Q_{i j}=Q_{i 1}+h_{i j}, \tilde{C}_{J_{i}}^{1,0}\left(Q_{i 1}\right)=\prod_{1}^{J_{i}} \tilde{C}^{1,0}\left(Q_{i 1}\right)-$ Cartesian product of $J_{i}$ spaces $\tilde{C}^{1,0}\left(Q_{i 1}\right)$.

Denote by $U_{i}^{-1}$ an isomorphism $U_{i}^{-1}: \tilde{C}_{J_{i}}^{1,0}\left(Q_{i 1}\right) \rightarrow \tilde{C}^{1,0}\left(\bigcup_{j=1}^{J_{i}} Q_{i j}\right)$ that is reverse to $U_{i}$. Using the isomorphism $U_{i}$, taking into account condition (6) and permutability of $U_{i}$ and $\partial / \partial t$, we obtain from equation (4)

$$
U_{i} R_{1 Q} U_{i}^{-1} U_{i} \frac{\partial x}{\partial t}=U_{i} R_{2 Q} U_{i}^{-1} U_{i} g(x)+U_{i} f, \quad\left((t, s) \in Q_{i 1}\right) .
$$

Denote vector-valued function $U_{i} x$ by $x^{i}, U_{i} g(x)$ by $\tilde{g}\left(x^{i}\right)$ and $U_{i} f$ by $f^{i}$. Using this notations, we can rewrite (7) as follows

$$
U_{i} R_{1 Q} U_{i}^{-1} \frac{\partial x^{i}}{\partial t}=U_{i} R_{2 Q} U_{i}^{-1} \tilde{g}\left(x^{i}\right)+f^{i}, \quad\left((t, s) \in Q_{i 1}\right) .
$$

The boundary conditions (3) can be rewritten in the same way 


$$
\left.x^{i}\right|_{t=0}=\varphi^{i}(s), \quad\left(s \in \Omega_{i 1}\right),
$$

where by $\varphi^{i}$ vector valued function $U_{i} \varphi$ is denoted.

Denote by $R_{\nu}^{i}, \nu=1,2, J_{i} \times J_{i}$ matrixes with elements $\left\{R_{\nu}^{i}\right\}_{k l}$, $\left\{R_{\nu}^{i}\right\}_{k l}(t, s)=a_{\nu}^{h}\left(t, s+h_{i k}\right)$, where $h=h_{i l}-h_{i k},(t, s) \in Q_{i 1}$.

Lemma 1. The operator $R_{\nu Q}^{i}=U_{i} R_{\nu Q} U_{i}^{-1}: \tilde{C}_{J_{i}}^{1,0}\left(Q_{i 1}\right) \rightarrow \tilde{C}_{J_{i}}^{1,0}\left(Q_{i 1}\right), \nu=$ $1,2, i=1, \ldots, I$ is the operator of multiplication by the matrix $R_{\nu}^{i}$.

The proof follows from lemma 8.6, ch. II, [5].

Theorem 1. Let $x$ be the solution to problem (4), (5), then for $i=1, \ldots, I$ vector-valued function $x^{i}=U_{i} x$ is the solution to the following problem

$$
\begin{gathered}
R_{1}^{i} \frac{\partial x^{i}}{\partial t}=R_{2}^{i} \tilde{g}\left(x^{i}\right)+f^{i}, \quad\left((t, s) \in Q_{i 1}\right), \\
\left.x^{i}\right|_{t=0}=\varphi^{i}(s), \quad\left(s \in \Omega_{i 1}\right),
\end{gathered}
$$

and if vector-valued function $x^{i}$ is the solution to problem (10), 11), then

$$
x(t, s)=U_{i}^{-1} x^{i}, \quad(t, s) \in\left(\bigcup_{j=1}^{J_{i}} Q_{i j}\right), \quad i=1, \ldots, I,
$$

is the solution to problem (4), (5).

Note that functions $R_{\nu}^{i}, \tilde{g}, f^{i}, \varphi^{i}$ are supposed to provide the existence and uniqueness of the solution to problem (10), (11) that is a continuously differentiable function with respect to $t$ and piecewise continuous function with respect to $s$, i.e. $x^{i} \in \tilde{C}_{J_{i}}^{1,0}\left(Q_{i 1}\right)$.

Proof. To begin the proof recall that the solution to problem (10)-(11) is a function that satisfies equation (10) and boundary condition (11) for almost all $s \in \Omega_{i 1}$.

Suppose $x$ is the solution to problem (4), (15). Since equation (17) is equivalent to equation (4) it follows that vector-valued function $x^{i}$ that is equal to $U_{i} x$ satisfies equation (8). Taking into account the above notations we obtain that vector-valued function $x^{i}$ satisfies equation (9). Finally, Lemma implies that $x^{i}$ satisfies equation (10).

Analogous reasoning implies that if $x$ satisfies initial condition (5) then vector-valued function $x^{i}$ satisfies initial conditions (11) for almost all $s$. Thus vector-valued function $x^{i}$ is the solution to problem (10), (11).

Now, suppose that vector-valued function $x^{i}(t)$ is the solution to problem (10), (11). It is clear that if $R_{\nu}^{i}(t, s), f^{i}(t, s), \varphi^{i}(s)$ are defined and continuous for some $s \in \Omega_{i j}$ then $x(t, s)$ specified in the statement of the theorem is defined, continuous with respect to $\mathrm{s}$ and continuously differentiable with respect to $t$ for 
the same $s$. Thus relation (6) implies that $x(t, s)$ has the same properties almost everywhere in $Q$, i.e. $x \in \widetilde{C}^{1,0}(Q)$.

Since $x^{i}, i=1, \ldots, I$ satisfies equation (10) then due to the Lemma it satisfies equation (8). The latter yield that $x(t, s)$ defined in the statement of the theorem satisfies equation (4) almost for all $s \in \Omega$. The same speculations show that $x$ satisfies initial condition (5) almost for all $s \in \Omega$. Thus, $x(t, s)$ is the solution to problem (4), (5).

Note to conclude that if $f(t, s), \varphi(s), a_{\nu}^{h}(t, s), \nu=1,2, h \in H$, are continuous functions with respect to $s$ for all $s \in \Omega$ may be except for points of the set $\left\{s \in \partial \Omega_{i j}, i=1, \ldots, I, j=1, \ldots, J_{i}\right\}$ then the solution to problem (4), (5) is a continuous function with respect to $s$ for all $s \in \Omega$ may be except for points of the same set. It worth adding that generally continuity of $f(t, s), \varphi(s), a_{\nu}^{h}(t, s)$ in $Q$ does not yield continuity with respect to $s$ of the solution to problem (4), (5).

\section{The Best Argument of the Problem}

To solve the problem of choosing the best argument of problem (4), (5) (the argument of differentiation), we exploit the method of the continuation of a solution. Theorem 1 implies that it is equivalent to seek the best argument of problem (10), (11).

Suppose matrixes $R_{1}^{i}, i=1, \ldots, I$, are not singular at $(t, s) \in Q_{i 1}$. Hence we can transform system (10), (11) to the normal form considering variable $s$ as a parameter.

$$
\begin{gathered}
\frac{d x^{i}}{d t}=\left(R_{1}^{i}(t, s)\right)^{-1} R_{2}^{i}(t, s) \tilde{g}\left(x^{i}\right)+\left(R_{1}^{i}(t, s)\right)^{-1} f^{i}(t, s), \\
x^{i}(0)=\varphi^{i}(s), \\
\left(t \in(0, T), s \in \Omega_{i 1}\right) .
\end{gathered}
$$

Assume that an integral of problem (12), (13) is given by

$$
F\left(x_{1}^{i}(t, s), \ldots, x_{r}^{i}(t, s), t\right)=0
$$

such that

$$
F\left(\varphi_{1}^{i}(s), \ldots, \varphi_{r}^{i}(s), 0\right)=0, s \in \Omega_{i 1},
$$

where $x_{l}^{i}$ are components of $x^{i}, \varphi_{l}^{i}-\varphi^{i}, F: \mathbb{R}^{r+1} \rightarrow \mathbb{R}^{r}$ (recall $r=J_{i}$ ), defines a smooth integral curve of the problem in $\mathbb{R}^{r+1}$ almost for every $s \in \Omega_{i 1}$.

We shall handle a process of this curve construction as a process of solving system (14), where $t$ is the argument of the problem and $s$ is regarded as a parameter, $s \in \Omega_{i 1}$. Also we'll investigate this system by the continuation method treating initial value problem (12), (13) as the Cauchy problem for continuation equations for the solution of system (14) with respect to parameter $t$. Such an approach has been used to solve the Cauchy problem for a system of ordinary differential equation [6] and delayed differential equation [7], [8]. 
Theorem 2. To transform the Cauchy problem (12), 13) to the best argument it is necessary and sufficient to choose the arc length $\lambda$ measured along the integral curve of this problem as a new independent variable for any particular $s \in \Omega_{i 1}$. After transformation problem (12), (13) takes the form

$$
\begin{array}{cc}
\frac{d x^{i}}{d \lambda}= \pm \frac{G^{i}\left(t, s, x^{i}\right)}{\sqrt{1+\left(G^{i}, G^{i}\right)}}, & x^{i}(0)=\varphi^{i}(s), \\
\frac{d t}{d \lambda}= \pm \frac{1}{\sqrt{1+\left(G^{i}, G^{i}\right)}}, & t(0)=0, \\
i=1, \ldots, I, \quad t \in(0, T), \quad s \in \Omega_{i 1} .
\end{array}
$$

Here by $(\cdot, \cdot)$ inner product in $\mathbb{R}^{r}$ and by $G^{i}=\left(G_{1}, \ldots, G_{r}\right)$ vector of righthand side functions of system (12) are denoted. Choice of plus or minus sign defines the direction of moving along the integral curve of the problem.

We call an argument to be the best if it provides the linear system of continuation equations whose matrix is the Jacobi matrix of system (14) with the largest possible condition number at any step of integration process.

Proof. We'll continue a solution to problem (12), (13) locally, in a small neighborhood of any point of the problem integral curve. Suppose that $t$ and all components of $x^{i}$ are functions of $\mu$ such that

$$
\Delta \mu=\sum_{l=1}^{r} c_{l} \Delta x_{l}^{i}+c_{r+1} \Delta t
$$

Here $\Delta x_{l}^{i}$ and $\Delta t$ are increments of corresponding variables, $c_{l}(l=1, \ldots, r+1)$ are components of the unit vector defining a direction in which $\mu$ is measured.

Having differentiated equation (14) with respect to $\mu$ as a composite function, we obtain

$$
\sum_{l=1}^{r} F_{k, x_{l}^{i}} x_{l, \mu}^{i}+F_{k, t} t_{, \mu}=0, \quad k=1, \ldots, r
$$

where the following notations were used

$$
x_{l, \mu}^{i}=\frac{d x_{l}^{i}}{d \mu}, \quad t_{, \mu}=\frac{d t}{d \mu}, \quad F_{k, x_{l}^{i}}=\frac{\partial F_{k}}{\partial x_{l}^{i}}, \quad F_{k, t}=\frac{\partial F_{k}}{\partial t} .
$$

Assuming nonsingularity of matrix $\left(F_{k, x_{l}^{i}}\right)$, we obtain from (18) and (12)

$$
\frac{d x_{l}^{i}}{d t}=-\left(F_{k, x_{l}^{i}}\right)^{-1}\left(F_{1, t}, \ldots, F_{r, t}\right)^{T}=G_{l}^{i} .
$$

Hence, equations (18) take the form

$$
x_{l, \mu}^{i}-G_{l}^{i} t_{, \mu}=0, \quad l=1, \ldots, r .
$$


Thus (19) implies that equations (12) multiplied by $d t$ and divided by $d \mu$ can be used as continuation equations.

To obtain $(r+1)$-th continuation equation one has to divide (17) by $\Delta \mu$ and then pass to limit as $\Delta \mu \rightarrow 0$.

$$
\sum_{l=1}^{r} c_{l} x_{l, \mu}^{i}+c_{r+1} t_{, \mu}=1 .
$$

Thus (19) and (20) constitute the system of continuation equations.

It has been proved (see [9]) that the transformation of this system to the Cauchy normal form is best conditioned iff $c_{l}=x_{l, \lambda}^{i}, l=1, \ldots, r, c_{r+1}=t_{, \lambda}$. This means that the arc length measured along the integral curve of problem (12), (13) is chosen as $\mu$. Hence, system (19), (20) can be rearranged

$$
\left\{\begin{array}{c}
x_{, \lambda}^{i}-G^{i} t_{, \lambda}=0 \\
\left(x_{l, \lambda}^{i}, x_{l, \lambda}^{i}\right)+t_{, \lambda}^{2}=1 .
\end{array}\right.
$$

Solving (21) for derivatives and measuring $\lambda$ from initial point of (12), (13) due to independence of right-hand side functions of the system on $\lambda$, we come to the transformed problem that is of the form given by (15), (16).

Example 1. Let consider the problem

$$
x^{\prime}(t, s-1)+t x^{\prime}(t, s)+x^{\prime}(t, s+1)=1 / 3 x(t, s), \quad((t, s) \in(0, T) \times(0,2)),
$$

with boundary condition $x(t, s)=0,(t, s) \in(0, T) \times(\mathbb{R} \backslash(0,2))$, and initial condition $x(0, s)=\varphi(s),(s \in(0,2))$.

In the above notations $I=1, J_{1}=2$. In this problem difference operator generates decomposition of $Q=(0, T) \times(0,2)$ into subdomains $Q_{11}=(0, T) \times$ $(0,1)$ and $Q_{12}=(0, T) \times(1,2)$. Functions $x^{i}$ and $\varphi^{i}$ correspond to each of these subdomains.

Define matrixes $R_{1}^{1}, R_{2}^{1}$

$$
R_{1}^{1}=\left(\begin{array}{cc}
t & 1 \\
1 & t
\end{array}\right), \quad R_{2}^{1}=\left(\begin{array}{cc}
1 / 3 & 0 \\
0 & 1 / 3
\end{array}\right) .
$$

Calculating a matrix inverse to $\left(R_{1}^{1}\right)^{-1}$ and denoting $x^{i}$ by $\left(x_{1}, x_{2}\right)^{T}$, we bring the problem to the form of (12), (13).

$$
\left(\begin{array}{l}
x_{1}^{\prime} \\
x_{2}^{\prime}
\end{array}\right)=\frac{1}{t^{2}-1}\left(\begin{array}{cc}
t & -1 \\
-1 & t
\end{array}\right)\left(\begin{array}{cc}
1 / 3 & 0 \\
0 & 1 / 3
\end{array}\right)\left(\begin{array}{l}
x_{1} \\
x_{2}
\end{array}\right) .
$$

or equivalently:

$$
\begin{aligned}
x_{1}^{\prime} & =\frac{1 / 3 t x_{1}}{t^{2}-1}-\frac{1 / 3 x_{2}}{t^{2}-1}, & x_{1}(0) & =\varphi_{1}(s), \\
x_{2}^{\prime} & =\frac{-1 / 3 x_{1}}{t^{2}-1}+\frac{1 / 3 t x_{2}}{t^{2}-1}, & x_{2}(0) & =\varphi_{2}(s) .
\end{aligned}
$$


The solution to this problem

$$
\begin{aligned}
& x_{1}=C_{1}(t+1)^{1 / 3}+C_{2}(t-1)^{1 / 3}, \\
& x_{2}=C_{1}(t+1)^{1 / 3}-C_{2}(t-1)^{1 / 3},
\end{aligned}
$$

where $C_{1}=\left(\varphi_{1}(s)+\varphi_{2}(s)\right) / 2, C_{2}=\left(\varphi_{1}(s)-\varphi_{2}(s)\right) / 2$ are obtained from the initial condition. As for the solution to the original problem, it has the form $x(t, s)=x_{i}(t, s),(t, s) \in Q_{1 i}, i=1,2$. It is evident that despite the right-hand side functions of system of differential equations are unbounded in a neighbourhood of $t= \pm 1$ the solution to the problem is a continuous function of $t$.

Having transformed the problem to the best argument, we obtain

$$
\begin{array}{cc}
\frac{d x_{1}}{d \lambda}=\frac{f_{1}}{\sqrt{\left(t^{2}-1\right)^{2}+f_{1}^{2}+f_{2}^{2}}},\left.\quad x_{1}\right|_{\lambda=0}=\varphi_{1}(s), \\
\frac{d x_{2}}{d \lambda}=\frac{f_{2}}{\sqrt{\left(t^{2}-1\right)^{2}+f_{1}^{2}+f_{2}^{2}}},\left.\quad x_{2}\right|_{\lambda=0}=\varphi_{2}(s), \\
\frac{d t}{d \lambda}=\frac{1}{\sqrt{\left(t^{2}-1\right)^{2}+f_{1}^{2}+f_{2}^{2}}},\left.\quad t\right|_{\lambda=0}=0,
\end{array}
$$

where $f_{1}=1 / 3\left(t x_{1}-x_{2}\right), f_{2}=-1 / 3\left(x_{1}-t x_{2}\right)$.

Note that right-hand side functions of the transformed system are continuous and uniformly bounded functions of $t$. It yields that the solution to the problem is a continuously differentiable function of $\lambda$.

Numerical tests demonstrate that unboundedness of the right-hand side functions of a system of differential equations may make impossible the implementation of standard numerical integration procedures. Preliminary transformation of a system to the best argument affords to avoid similar difficulties.

\section{References}

1. Kamenskii, G.A., Myshkis, A.D.: On the mixed type functional differential equations. Nonlinear Analysis, TMA. Vol. 30.5 (1997) 2577-2584

2. Kamenskii, G.A.: A review of the theory of mixed functional differential equations. Problems of Nonlinear Analysis in Engineering Systems. Vol. 2. 8 (1998) 1-16

3. Kamenskii, G.A.: Boundary value problems for difference-differential equations arising from variational problems. Nonlinear Analysis, TMA. Vol. 18. 8 (1992) 801-813

4. Kopylov, A.V.: Finite difference method for the numerical solution of boundary value problem for mixed difference-differential equation. Sib. J. Numer. Math. Vol. 3. 4 (2000) 345-355 (Russian)

5. Skubachevskii, A.L.: Elliptic functional differential equations and applications. Birkhauser, Basel Boston Berlin (1997)

6. Kuznetsov, E.B., Shalashilin, V.I.: The Cauchy problem as a problem of continuation with respect to the best parameter. Differ. Eq. Vol. 30. 6 (1994) 893-898

7. Kuznetsov, E.B.: Transformation delayed differential equations to the best argument. Mat. Zametki Vol. 63. 1 (1998) 62-68 
8. Kopylov, A.V., Kuznetsov, E.B.: An approach to the numerical integration of the Cauchy problem for Delay Differential Equations. Comput. Math. Math. Phys. Vol. 41. 10 (2001) 1470-1479

9. Kuznetsov, E.B., Shalashilin, V.I.: Method continuation of a solution with respect to the best parameter and the best parameterization in applied mathematics and mechanics. Editorial URSS, Moscow (1999) (Russian) 